\title{
CORRIGENDUM
}

\section{Long-term treatment of phenylketonuria with a new medical food containing large neutral amino acids}

D Concolino, I Mascaro, MT Moricca, G Bonapace, K Matalon, J Trapasso, G Radhakrishnan, C Ferrara, R Matalon and P Strisciuglio

European Journal of Clinical Nutrition (2017) 71, 1027; doi:10.1038/ejcn.2017.98

Correction to: European Journal of Clinical Nutrition (2017); 71 (1); 51-55; doi: 10.1038/ejcn.2016.166; published online 14th September 2016

Since the publication of the article, the authors have noticed an error in Table 1. The corrected table can be found below.

The authors apologise for any inconvenience caused by this error.

Table 1. Composition of PheLNAA for $100 \mathrm{~g}$ powder

\begin{tabular}{|c|c|c|c|c|c|}
\hline \multirow[t]{3}{*}{ Energy } & kcal & 293.50 & Vitamins & & \\
\hline & $\mathrm{kJ}$ & 1241.26 & A & $\mathrm{mcg}$ & 1300 \\
\hline & & & $D_{3}$ & mcg & 15.5 \\
\hline Protein equivalent & $\mathrm{g}$ & 48.94 & $\mathrm{E}$ & $\mathrm{mg}$ & 20 \\
\hline Carbohydrates & $g$ & 15.74 & $\mathrm{~K}_{1}$ & $\mathrm{mcg}$ & 113 \\
\hline Sugars & $\mathrm{g}$ & 12.46 & $C$ & $\mathrm{mg}$ & 100 \\
\hline Fats & $\mathrm{g}$ & 2.58 & $\mathrm{~B}_{1}$ & $\mathrm{mg}$ & 1.5 \\
\hline \multirow[t]{3}{*}{$\mathrm{DHA}$} & $\mathrm{mg}$ & 610 & $\mathrm{~B}_{2}$ & $\mathrm{mg}$ & 1.8 \\
\hline & & & $\mathrm{B}_{6}$ & $\mathrm{mg}$ & 5.4 \\
\hline & & & $\mathrm{B}_{12}$ & $\mathrm{mcg}$ & 9 \\
\hline Amino Acids & $\mathrm{g}$ & 61.73 & Biotin & mcg & 150 \\
\hline Arginine & $\mathrm{g}$ & 2.79 & Choline & $\mathrm{mg}$ & 650 \\
\hline Aspartic Acid & $\mathrm{g}$ & 2.36 & Inositol & $\mathrm{mg}$ & 125 \\
\hline Glutamine & $\mathrm{g}$ & 3.52 & Niacin & $\mathrm{mg}$ & 20 \\
\hline Isoleucine & $\mathrm{g}$ & 3.33 & Folic acid & $\mathrm{mcg}$ & 750 \\
\hline Leucine & $\mathrm{g}$ & 9.79 & Pantothenic acid & $\mathrm{mg}$ & 8.8 \\
\hline Lysine & $\mathrm{g}$ & 5.70 & Minerals & & \\
\hline Methionine & $g$ & 0.97 & Calcium & $\mathrm{mg}$ & 1600 \\
\hline Proline & $\mathrm{g}$ & 3.45 & Chromium & mcg & 40 \\
\hline Threonine & $\mathrm{g}$ & 3.15 & Copper & $\mathrm{mg}$ & 1.5 \\
\hline Tryptophan & $\mathrm{g}$ & 3.33 & lodine & $\mathrm{mcg}$ & 200 \\
\hline Tyrosine & $\mathrm{g}$ & 18.79 & Iron & $\mathrm{mg}$ & 20 \\
\hline Valine & $\mathrm{g}$ & 3.33 & Magnesium & $\mathrm{mg}$ & 500 \\
\hline \multirow[t]{7}{*}{ Hystidine } & $\mathrm{g}$ & 1.21 & Manganese & $\mathrm{mg}$ & 3 \\
\hline & & & Molybdenum & $\mathrm{mcg}$ & 90 \\
\hline & & & Phosphorous & $\mathrm{mg}$ & 1250 \\
\hline & & & Selenium & $\mathrm{mcg}$ & 75 \\
\hline & & & Sodium & $\mathrm{mg}$ & 40 \\
\hline & & & Zinc & $\mathrm{mg}$ & 15 \\
\hline & & & Lutein & $\mathrm{mg}$ & 12 \\
\hline
\end{tabular}

(i) This work is licensed under a Creative Commons Attribution 4.0 International License. The images or other third party material in this article are included in the article's Creative Commons license, unless indicated otherwise in the credit line; if the material is not included under the Creative Commons license, users will need to obtain permission from the license holder to reproduce the material. To view a copy of this license, visit http://creativecommons.org/licenses/ by/4.0/

(c) The Author(s) 2017 